\title{
A SHARP COUNTEREXAMPLE ON THE REGULARITY OF $\Phi$-MINIMIZING HYPERSURFACES
}

\author{
FRANK MORGAN
}

A standard problem in the calculus of variations seeks a hypersurface $S$ of least area bounded by a given $(n-2)$-dimensional compact submanifold of $\mathbf{R}^{n}$. More generally, given any smooth norm $\Phi$ on $\mathbf{R}^{n}$, seek to minimize

$$
\Phi(S)=\int_{S} \Phi(\mathbf{n})
$$

where $\mathbf{n}$ is the unit normal vector to $S$. Think of the integrand $\Phi$ as assigning a cost or energy to each direction. We assume that $\Phi$ is elliptic (uniformly convex), the standard hypothesis for regularity.

Geometric measure theory (cf. [M, Chapters 5, 8], [F 1, 5.1.6, 5.4.15]) guarantees the existence of a (possibly singular) $\Phi$-minimizing hypersurface with given boundary. For the case of area $(\Phi(\mathbf{n})=1)$, area-minimizing hypersurfaces are regular embedded manifolds up through $\mathbf{R}^{7}$, but sometimes have singularities in $\mathbf{R}^{8}$ and above. For general elliptic $\Phi$, a result of Almgren, Schoen, and Simon [Alm S S, Theorem II.7] guarantees regularity up through $\mathbf{R}^{3}$, but there were no examples of singularities below $\mathbf{R}^{8}$. We establish the sharpness of the Almgren-Schoen-Simon regularity result by giving a singular $\Phi$-minimizing hypersurface in $\mathbf{R}^{4}$.

The surface is the cone $C$ over the Clifford torus $S^{1} \times S^{1} \subset$ $\mathbf{R}^{2} \times \mathbf{R}^{2}$ :

$$
C=\left\{(x, y) \in \mathbf{R}^{2} \times \mathbf{R}^{2}:|x|=|y| \leq 1\right\} .
$$

The norm $\Phi$ depends smoothly on $\theta=\tan ^{-1}(|y| /|x|)$ alone, so that we may view $\Phi$ as a norm on $\mathbf{R}^{2}$. The unit $\Phi$-ball is pictured in Figure 1. Any smooth, symmetric, uniformly convex approximation of the square will do. Note that $\Phi$ is smaller (say 1) on

Received by the editors February 10, 1989; in revised form September 25, 1989. 1980 Mathematics Subject Classification (1985 Revision). Primary 49F22.

Key words and phrases. Elliptic integrand, calibration, minimizing hypersurface, Wulff crystal. 


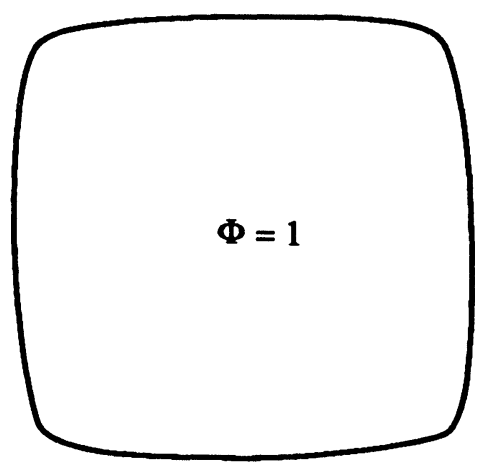

Figure 1. The unit $\Phi$-ball. The smallness of $\Phi$ in the diagonal directions helps to make the cone $C \Phi$ minimizing.

the normals to diagonal directions, which occur in the cone $C$, so

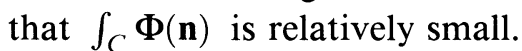

The unit ball of the norm dual to $\Phi$, called the Wulff crystal $W(\Phi)$, is pictured in Figure 2. The Wulff crystal itself solves an important problem: its boundary surface $S$ minimizes $\Phi(S)$ for fixed volume enclosed (cf. [T, §1]). In nature $\Phi(S)$ represents the surface energy of a crystal, and the Wulff crystal $W(\Phi)$ gives the shape which a fixed volume of material assumes to minimize surface energy. The Wulff crystal of our norm $\Phi$ resembles a pivalic acid crystal (see Figure 3).

The Proof. The proof that the cone $C$ over $S^{1} \times S^{1} \subset \mathbf{R}^{4}$ is $\Phi$-minimizing employs the "method of calibrations" (cf. [HL, Introduction]). One must produce a closed differential 3-form or "calibration" $\varphi$ such that for any point $p$ and unit 3-plane $\xi$, with unit normal $* \xi$,

$$
\langle\xi, \varphi(p)\rangle \leq \Phi(* \xi),
$$

with equality whenever $\xi$ is the oriented unit tangent to $C$ at $p$. Then if $S$ is any other surface with the same boundary,

$$
\Phi(C)=\int_{C} \varphi=\int_{S} \varphi \leq \Phi(S),
$$

so that $C$ is $\Phi$-minimizing.

Finding a calibration $\varphi$ remains an art, not a science. Our calibration is

$$
\varphi=\left(\sin ^{2} 2 \theta d r+\sin 4 \theta r d \theta\right) \wedge d \theta_{1} \wedge d \theta_{2},
$$




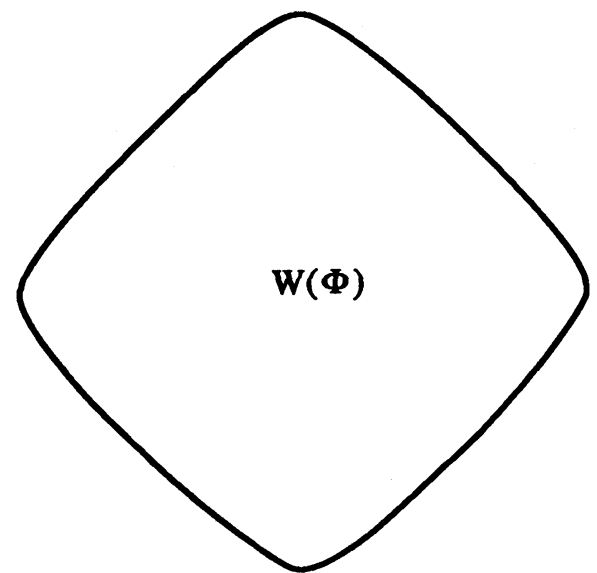

Figure 2. The Wulff crystal $W(\Phi)$ may be defined as the unit ball for the norm dual to $\Phi$. For fixed volume, $W(\Phi)$ has the least surface energy measured by $\Phi$.

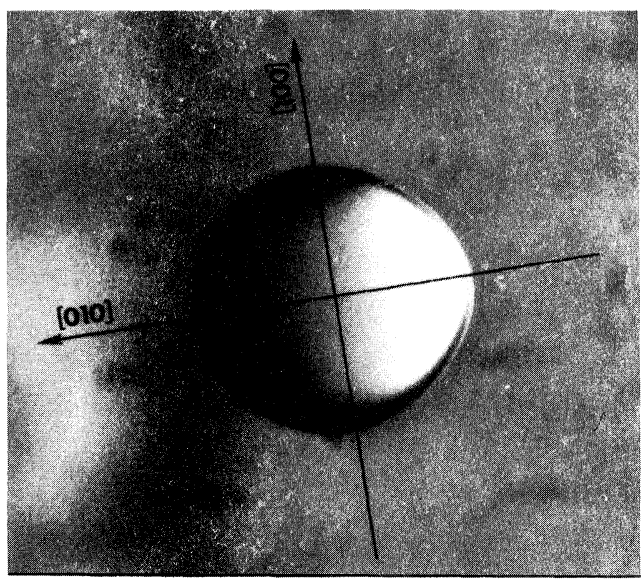

Figure 3. The pivalic acid crystal resembles the Wulff crystal $W(\boldsymbol{\Phi})$ of $\boldsymbol{\Phi}$ [GS].

where $r^{2}=|x|^{2}+|y|^{2}, \theta=\tan ^{-1}(|y| /|x|), \theta_{1}=\arg x, \theta_{2}=\arg y$.

It resembles the 7-form of $H$. Federer's proof [F2, $\S 6.3]$ after $H$. B. Lawson [L, §5] that the cone over $\mathbf{S}^{3} \times \mathbf{S}^{3}$ is area-minimizing. At any point in our cone $C, \theta=\pi / 4$, and $\varphi(\pi / 4)=d r \wedge d \theta_{1} \wedge d \theta_{2}$ is precisely dual to each unit tangent $\xi_{0}$ to $C$. Hence

$$
\langle\xi, \varphi(p)\rangle \leq 1 \leq \Phi(* \xi),
$$


with equality whenever $\xi=\xi_{0}$. Thus (1) holds at points $p \in C$. Unfortunately, for $p \notin C$ (for example $\theta=\pi / 8$ ), the $\sin 4 \theta$ term, which is necessary to make $\varphi$ closed, tends to make $\varphi$ big. In order for (1) to hold, the largeness of $\varphi(\pi / 8)$ must be somehow compensated for by the largeness of $\Phi(\pi / 8)$.

Establishing the estimate (1) at all points almost always is a main difficulty.

For the case of area, the right-hand side is 1 , and the estimate becomes $|\varphi(p)| \leq 1$, independent of $\xi$. For a general integrand $\Phi$, the estimate involves both $p$ and $\xi$. This difficulty explains why calibrations have not been applied specifically to integrands other than area before.

We handle this difficulty with a lemma that associates with $\varphi$ the function on unit vectors

$$
\begin{aligned}
& G(w)=\sup \{|\varphi(p)|: w \text { is the oriented unit normal } \\
& \text { to the }(n-1) \text {-plane dual to } \varphi(p)\} .
\end{aligned}
$$

The lemma says that the desired estimate (1) holds if the graph of $G$ lies inside the Wulff crystal $W(\Phi)$, thus reducing the required estimate to a single parameter.

\section{ACKNOWLEDGMENTS}

I would like to thank Williams students Ken Hodges, Rajiv Kochar, Lisa Kuklinski, Adam Levy, Zia Mahmood, and Kob Pootrakool for their help in this research. An improvement from $\mathbf{R}^{6}$ to $\mathbf{R}^{4}$ was obtained while I was visiting J. M. Coron at the Universite de Paris-Sud, Centre d'Orsay, in June, 1988.

This work was partially supported by a grant from the National Science Foundation.

\section{REFERENCES}

[Alm S S] F. J. Almgren, Jr., R. Schoen and L. Simon, Regularity and singularity estimates on hypersurfaces minimizing elliptic variational integrals, Acta Math. 139 (1977), 217-265.

[F 1] H. Federer, Geometric measure theory, Springer-Verlag, New York, 1969.

[F 2] _ Real flat chains, cochains, and variational problems, Indiana Univ. Math. J. 24 (1974), 351-407.

[GS] M. E. Glicksman and N. B. Singh, Microstructural scaling laws for dentritically solidified aluminum alloys, Special Technical Pub. 890, Amer. Soc. for Testing and Materials, Philadelphia, 1986, pp. 44-61.

[HL] R. Harvey and H. B. Lawson, Jr., Calibrated geometries, Acta Math. 148 (1982), 47-157. 
[L] H. B. Lawson, Jr., The equivariant plateau problem and interior regularity, Trans. Amer. Math. Soc. 173 (1972), 231-247.

[M] F. Morgan, Geometric measure theory: A beginner's guide, Academic Press, New York, 1988.

[T] J. E. Taylor, Crystalline variational problems, Bull. Amer. Math. Soc. 84 (1978), 568-588.

Department of Mathematics, Williams College, Williamstown, MasSACHUSETTS 01267 
